\title{
Studies of the Articular Cartilage Proteoglycan Aggrecan in Health and Osteoarthritis

\author{
Evidence for Molecular Heterogeneity and Extensive Molecular Changes in Disease
}

\author{
Geihan Rizkalla, * Agnes Reiner, * Earl Bogoch, ${ }^{\star}$ and A. Robin Poole* \\ *Joint Diseases Laboratory, Shriners Hospital for Crippled Children, Division of Surgical Research, Department of Surgery, \\ McGill University, Montreal, Quebec, Canada, H3G 1A6; and ${ }^{\ddagger}$ Department of Orthopaedic Surgery, \\ Wellesley Hospital, University of Toronto, Toronto, Ontario, Canada, M4Y $1 \mathrm{~J} 3$
}

\begin{abstract}
Changes in the structure of the proteoglycan aggrecan (PG) of articular cartilage were determined immunochemically by RIA and gel chromatography and related to cartilage degeneration documented histologically by the Mankin grading system. Monoclonal antibodies to glycosaminoglycan epitopes were used. In all cartilages, three chondroitin sulfate (CS)-rich populations of large size were observed in addition to a smaller keratan sulfate (KS)-rich population. In grades 7-13 OA cartilages (phase II), molecules were significantly larger than the equivalent molecules of grades 2-6 (phase I). CS chain lengths remained unchanged. In most OA cartilages, a CS epitope 846 was elevated in content, this being most marked in phase II (mean: fivefold). Loss of uronic acid, KS, and hyaluronic acid were only pronounced in phase II OA because of variations in normal contents. Aggregation of PG was unchanged (50-60\%) or reduced in OA cartilages, but molecules bearing epitope 846 exhibited almost complete aggregation in normal cartilages. This study provides evidence for the capacity of OA cartilage to synthesize new aggrecan molecules to replace those damaged and lost by disease-related changes. It also defines two phases of PG change in OA: an early predominantly degenerate phase I followed by a net reparative phase II accompanied by net loss of these molecules. (J. Clin. Invest. 1992.90:2268-2277.) Key words: cartilage $\bullet$ proteoglycan • osteoarthritis • aggrecan • immunochemistry
\end{abstract}

\section{Introduction}

Osteoarthritis is a degenerative joint disease that involves impairment and eventually loss of joint function. It is characterized by extensive degeneration of the articular cartilages in both large and small joints, usually in the presence of low level intraarticular inflammation (1).

The articular cartilage of a diarthrodial joint is composed of an extensive network of collagen fibrils that provide cartilage

Address all correspondence to Dr. A. Robin Poole, Shriners Hospital for Crippled Children, Division of Surgical Research, Department of Surgery, McGill University, 1529 Cedar Ave., Montreal, Quebec, Canada, H3G 1 A6.

Geihan Rizkalla's current address is Department of Psychiatry, Royal Victoria Hospital, McGill University.

Received for publication 26 February 1992 and in revised form 31 July 1992

J. Clin. Invest.

(C) The American Society for Clinical Investigation, Inc.

$0021-9738 / 92 / 12 / 2268 / 10 \$ 2.00$

Volume 90, December 1992, 2268-2277 with its tensile strength and stiffness $(2,3)$. This is composed predominantly of type II collagen. Type XI collagen is present within the fibril (4). On its surface, covalently attached to type II collagen in the nonhelical telopeptide regions, resides type IX collagen $(5,6)$. Interacting with this network, either directly or indirectly, is a "mesh work" of high molecular weight hyaluronic acid (HA) 1 (7). A large proteoglycan (PG), called aggrecan, which on synthesis has a $2-4 \times 10^{6} M_{\mathrm{r}}$, binds to HA through an amino-terminal G1 globular domain (8). Each attachment is stabilized by a single link protein $(9,10)$ that shares sequence homology with the $\mathrm{G} 1$ domain $(11,12)$.

The core protein of aggrecan contains a second globular domain called G2 which has considerable homology with G1 (12): its function is unknown. Between G2 and another globular domain called G3 $(12,13)$ situated at the carboxyl-terminal, there are the glycosaminoglycan attachment regions $(12,13)$. Adjacent to $G 2$ there is a keratan sulfate (KS) attachment region composed of repeating hexapeptide motif and containing up to $30 \mathrm{KS}$ chains $(13,14)$. Between this region and G3 there is a high concentration of up to $100 \mathrm{CS}$ chains (13). There is evidence from studies of human aggrecan, based on core protein structure, that this region may be composed of two subdomains called CS-1 and CS-2 (13). The G3 domain has been shown to have lectin-like properties, since it binds to galactosyl residues (15). This property may permit interactions with molecules in the extracellular matrix.

Antibodies have been prepared to unsaturated sulfated and unsulfated disaccharides of chondroitin sulfate (CS), some of which remain as stubs attached to the core protein of aggrecan after eliminative digestion of CS (16, 17). These antibodies react preferentially with the "stubs" that remain attached to core protein, and are sulfation specific (disaccharide chondroitin 4-sulfate stubs attached to core protein [ $\Delta$ diC4S-s], disaccharide chondroitin 6-sulfate stubs attached to core protein [ $\Delta$ diC6S-s], and disaccharide chondroitin stubs attached to core protein [ $\Delta$ diCOS-s] ). More recently, antibodies to native CS epitopes have been prepared $(16,18,19)$. These are usually most commonly found in fetal or embryonic tissues. The majority of the epitopes they recognize remain to be characterized. Antibodies to $\operatorname{KS}(20,21)$ have been shown to recognize highly sulfated domains on this glycosaminoglycan (22). Use of these antibodies in immunoassays permits the detection and measurement of PG and fragments thereof in cartilages in the presence of other macromolecules (17).

1. Abbreviations used in this paper: CS, chondroitin sulfate; $\Delta$ diC4S-s, disaccharide chrondroitin 4-sulfate stubs attached to core protein; $\Delta$ diC6S-s, disaccharide chondroitin 6-sulfate stubs attached to core protein; $\mathrm{GuCl}$, guanidinium chloride; $\mathrm{HA}$, hyaluronic acid; $\mathrm{KS}$, keratan sulfate; OA, osteoarthritis; PG, proteoglycans; UA, uronic acid. 
These proteoglycans constitute up to $10 \%$ of the wet weight of cartilage. Because of their high content of glycosaminoglycans, they become very hydrated and can absorb up to 50 times their weight of water. But this hydration is constrained by the tensile strength of the collagen network. This results in a swelling or osmotic pressure that endows cartilage with its compressive stiffness and hence its reversible deformability (23-25). As human articular cartilage ages, there is an accumulation of the G1 globular domain (26) and of smaller KS-rich aggrecan molecules $(27,28)$. These proteoglycan degradation products increase in content mainly in the deep zone $(28,29)$. The degradation probably occurs over a period of time in the extracellular matrix. Further evidence for extracellular degradation is provided by the progressive accumulation with aging of link protein of reduced size (30). This probably results in part from the extracellular action of proteinases, such as the metalloproteinase stromelysin (31).

There have been many studies of the PG aggrecan in osteoarthritic articular cartilages. These have sometimes produced contradictory results. Studies of molecular size and aggregation with HA invariably involved measurement of uronic acid or hexosamine content. These investigations concluded that there is either no change (32), a reduction $(33,34)$, or an increase (35) in molecular size in osteoarthritis (OA). Loss of aggregation in advanced disease has been reported (36), but others found no change in aggregation (32). There is, however, a reduction in KS relative to CS content (33). Synthesis is often increased in early degeneration and later decreased as cartilage is further destroyed $(37,38)$. These observations indicate that there are significant changes in this PG in OA, but the precise molecular events involved and implications of these changes are unclear, since these studies were made on many different specimens, concentrating on chemical analyses of the chondroitin sulfate of isolated high buoyant density PG. Moreover, in most of these studies, with a few exceptions in which synthesis was studied $(37,38)$, there was a failure to relate molecular changes to the different stages of degenerative change that Mankin et al. have described (38). Cartilages were usually inappropriately treated as a homogeneous group.

We therefore decided to use newly developed quantitative immunochemical assays for the study of these molecules in total cartilage extracts so that aggrecan size, heterogeneity, composition, degradation products, and aggregation could be clearly identified in normal and OA cartilages that had been age- and tissue-matched and histologically graded for degenerative changes. The study presented here provides evidence for even more molecular heterogeneity than has been previously recognized. It also reveals biosynthetic and degradative changes in OA that are indicative of an initial phase of net degradation followed by a phase of extensive replacement with new aggrecan molecules that are then partially degraded: Eventually, there is a net loss of these molecules in advanced disease.

\section{Methods}

Cartilage. Human articular cartilage that appeared macroscopically normal was obtained at autopsy within $12 \mathrm{~h}$ of death from femoral condyles of adults (ages 47-81 yr) with no evidence of joint trauma, connective tissue abnormality, or arthritic disease. The cartilage was frozen at $-20^{\circ} \mathrm{C}$ until processed. Human $\mathrm{OA}$ femoral condyles obtained at surgery for knee arthroplasty were frozen immediately after removal. The age range of the patients was 59-77 yr. Altogether, six normal and seven OA joints were studied for total contents, molecular sizes, and aggregation.

$1-\mathrm{cm}^{2}$ specimens of the whole depth of cartilage $(\sim 100-200 \mathrm{mg}$ in wet $w t$ ) from the distal weight bearing areas of the femoral condyles of normal and OA joints were removed, taking care to exclude underlying subchondral bone. In some of the normal and OA samples, two specimens were prepared at different sites of the weight-bearing region of the same femoral condyle to study variations within different sites of the same joint. Specimens were frozen sectioned at $20 \mu \mathrm{m}$ with a cryostat (Tissue TEK II; Miles Scientific Div., Miles Laboratories Inc., Naperville, IL) before extraction. Fetal, epiphyseal, and articular cartilages were obtained within $16 \mathrm{~h}\left(\right.$ at $\left.4^{\circ} \mathrm{C}\right)$ of therapeutic abortion.

Histology. A full depth sample adjacent to the specimen chosen for immunochemical and biochemical analyses was removed and fixed for $24 \mathrm{~h}$ in $10 \%$ formalin in $25 \mathrm{mM}$ sodium phosphate, $\mathrm{pH} 7.0$, for histology. After wax embedding, $6-\mu \mathrm{m}$ thick sections taken at two different levels perpendicular to the articular surface were cut and stained with hematoxylin and eosin or with safranin-O and fast green-iron hematoxylin. Sections were graded according to Mankin's histological grading system for OA cartilages (38). This method assigned scores to the intactness of the structure of the tissue, the sizes and grouping of the cells and the degree of safranin-O (proteoglycan) staining. The scores were totaled for each sample and a grade was assigned. Since sections were cut up to, but not including the tide mark, this was excluded from the grading. The maximum score possible, when tide mark is not included, is 13 .

Extraction of $P G$. The $20-\mu \mathrm{m}$ thick frozen sections from each sample were extracted with $30 \mathrm{vol}(\mathrm{wt} / \mathrm{vol})$ of $4 \mathrm{M}$ guanidinium chloride $(\mathrm{GuCl})$ in $100 \mathrm{mM}$ sodium acetate, $\mathrm{pH} 6.0$ (containing $1 \mathrm{mM}$ EDTA, $1 \mathrm{mM}$ iodoacetic acid, $1 \mathrm{mM}$ phenylmethylsulphonyl fluoride with 5 $\mu \mathrm{g} / \mathrm{ml}$ of pepstatin to inhibit metallo, cysteine, serine, and aspartate proteinases, respectively) at $4^{\circ} \mathrm{C}$ for $48 \mathrm{~h}$ with constant stirring. The PG extracts were clarified by centrifugation in a Sorval GLC-2B centrifuge at $3,500 \mathrm{rpm}(2,000 \mathrm{~g})$ for $15 \mathrm{~min}$ to remove any particulate material. Supernatant and pellet were stored separately at $-20^{\circ} \mathrm{C}$. Before assay for uronic acid (see below) pellets were rinsed with distilled water, dissolved in $2 \mathrm{ml} 7 \mathrm{~N} \mathrm{HCl}$ and incubated at $70^{\circ} \mathrm{C}$ for $20 \mathrm{~min}$.

Isolation and purification of proteoglycan standards for radioimmunoassays. Aggrecan monomer was prepared from extracts of fetal and adult cartilages ( $10 \mathrm{vol}$ of $4 \mathrm{M} \mathrm{GuCl}$ with proteinase inhibitors), by density gradient centrifugation in gradients of cesium chloride, as described previously (39): Fraction D1 of highest density (>1.54) was isolated and used as the source of aggrecan.

Hyaluronic acid. A radiometric assay was used as described by the manufacturer (Pharmacia Fine Chemicals, Uppsala, Sweden).

Uronic acid. This was determined by the carbazole reaction ( 40 ) on pellets (nonextractable) and after dialysis of extracts against deionized water.

Immunoassays. Radioimmunoassays were performed using antibodies to $\mathrm{KS}$ (AN9P1) $(21,27)$, and to unsaturated disaccharides produced by digestion of $C S$ with chondroitinase $A B C$, namely $\triangle$ diC4S-s monoclonal 6B (17) and $\Delta$ diC6S-s (monoclonal 3B3) (16). A mouse monoclonal antibody 846 was used that reacts with an as yet unidentified fetal-common epitope on the native CS of aggrecan (chondroitinase $\mathrm{ABC}$ labile) (Poole, A. R., and A. Reiner, unpublished observation) and not on core protein as previously concluded (18).

The radioimmunoassays were as described previously for AN9P1 (27), 6B (17), and $846(18)$. These used both ${ }^{125}$ I-labeled and unlabeled (for tracer and standard, respectively) human fetal ( 846 and $6 \mathrm{~B}$ ) and adult (AN9P1 and 3B3) human aggrecan. Chondroitinase ABCtreated aggrecan (17) was used for tracer and standards for assays with antibodies $6 \mathrm{~B}$ and $3 \mathrm{~B} 3$ to detect primarily unsaturated stubs of $\Delta$ diC4S-s and $\Delta$ diC6S-s, respectively, that remain bound to core protein after digestion with chondroitinase $\mathrm{ABC}$ (17) (Hirata, S., and A. R. Poole, unpublished observations). Intact proteoglycan (tracer and standard) was used for assays with antibodies AN9P1 and 846 for KS and CS epitope 846 , respectively. 
Table I. Summary of Radioimmunoassays with Different Antibodies

\begin{tabular}{|c|c|c|c|c|}
\hline Antibody and recognition site & $\begin{array}{l}\text { Standard and } \\
\text { sample treatments }\end{array}$ & $\begin{array}{l}\text { Radiolabled } \\
\text { proteoglycans }\end{array}$ & $\begin{array}{l}\text { Standard } \\
\text { proteoglycan }\end{array}$ & Precipitation step \\
\hline \multicolumn{5}{|l|}{ 3-B-3 } \\
\hline $\begin{array}{l}\text { Mouse IgM recognizes } \triangle \text { diC6S-s left } \\
\text { on core protein after chase } A B C \\
\text { treatment }\end{array}$ & $\begin{array}{l}\text { Chase } \mathrm{ABC} \text { and } \\
\text { SDS }\end{array}$ & $\begin{array}{l}{ }^{125} \mathrm{I}-\mathrm{HAPG} / \text { chase } \\
\text { ABC }\end{array}$ & HAPG & $\begin{array}{l}\text { R191 (rabbit anti-mouse } \\
\text { IgM) and protein A }\end{array}$ \\
\hline \multicolumn{5}{|l|}{846} \\
\hline $\begin{array}{l}\text { Mouse IgM recognizes CS epitope } \\
\text { expressed maximally on HFPG }\end{array}$ & SDS & ${ }^{125} \mathrm{I}-\mathrm{HFPG} /$ native & HFPG & $\begin{array}{l}\text { R191 (rabbit anti-mouse } \\
\text { IgM and protein A) }\end{array}$ \\
\hline \multicolumn{5}{|l|}{$6 \mathrm{~B}$} \\
\hline $\begin{array}{l}\text { Mouse IgG recognizes } \Delta \text { diC4S-s } \\
\text { stubs left on core protein after } \\
\text { chase } A B C \text { treatment }\end{array}$ & $\begin{array}{l}\text { Chase } \mathrm{ABC} \text { and } \\
\text { SDS }\end{array}$ & $\begin{array}{l}{ }^{125} \text { I-HFPG/chase } \\
\text { ABC }\end{array}$ & HFPG & Protein A \\
\hline \multicolumn{5}{|l|}{ AN9P1 } \\
\hline $\begin{array}{l}\text { Mouse IgG recognizes a KS-rich } \\
\text { PG, native }\end{array}$ & SDS & $\begin{array}{l}{ }^{125} \text { I-HAPG/ } \\
\text { native }\end{array}$ & HAPG & Protein A \\
\hline
\end{tabular}

Additional abbreviations used are chase ABC, chondroitinase ABC; HAPG and HFPG, human adult and fetal aggrecan, D1 preparation.

Wherever necessary, all samples were dialyzed before assay (by microdialysis with a 3,500 D cut off membrane) for $48 \mathrm{~h}$ at room temperature to remove $4 \mathrm{M} \mathrm{GuCl}$ ( $150 \mathrm{vol}$ of $200 \mathrm{mM}$ sodium acetate, $\mathrm{pH} 5.5$ containing $0.05 \%$ sodium azide). Subsequently, before assay, all samples $(100 \mu \mathrm{l})$ were treated with $100 \mu \mathrm{l} 200 \mathrm{mM}$ Tris/acetate, $\mathrm{pH} 7.5$, containing $0.04 \mathrm{U} / \mathrm{ml}$ chondroitinase $\mathrm{ABC}$ (ICN, Montreal), and were incubated for $6 \mathrm{~h}$ at $37^{\circ} \mathrm{C}$. At the end of incubation, $50 \mu \mathrm{l}$ of $0.125 \%$ SDS in $100 \mathrm{mM}$ Tris/acetate buffer, $\mathrm{pH} 7.6$, was added to give a final concentration of $0.025 \%$ SDS. This mixture was incubated at $80^{\circ} \mathrm{C}$ for 15 min to disaggregate any PG aggregates (17) to ensure that epitopes on PG molecules were maximally exposed to antibody. Human adult and PG used for standards were diluted in associative column buffer in the range $0.05-200 \mu \mathrm{g} / \mathrm{ml}$ and treated in the same way. Radioimmunoassay buffer contained $7.5 \mathrm{mM}$ potassium dihydrogen phosphate, $134.9 \mathrm{mM}$ disodium hydrogen phosphate at $\mathrm{pH} 8.0$, containing $0.1 \%$ BSA, $0.5 \%$ sodium deoxycholate, $0.25 \%$ Nonidet P-40 and $0.05 \%$ sodium azide. Results of all immunoassays were expressed in equivalents of intact adult or fetal PG. The use of both adult and fetal PG was necessitated by the fact that antibodies $6 \mathrm{~B}$ and 846 show minimal reactivity with adult proteoglycans, whereas AN9P1 and 3B3 exhibit minimal reactivity to fetal PG.

A summary of the antibodies used and main features of their assays is shown in Table I. The results of typical inhibition profiles are shown in Fig. 1.

\section{Gel chromatography to determine molecular sizes, aggregation with hyaluronic acid, and chondroitin sulfate chain length}

Dissociative conditions. To determine the PG monomer sizes in total extracts of cartilage samples, $500 \mu \mathrm{l}$ of extract in $4 \mathrm{M} \mathrm{GuCl}$ with proteinase inhibitors were chromatographed under dissociative conditions on Sepharose CL-2B $(100 \mathrm{~cm} \times 1.25 \mathrm{~cm}$ in diameter, $6 \mathrm{ml} / \mathrm{h}$ flow rate, $1 \mathrm{ml}$ fractions, Pharmacia Fine Chemicals, Montreal, Canada), eluting with $4 \mathrm{M} \mathrm{GuCl}, 50 \mathrm{mM}$ Tris $/ \mathrm{HCl}, \mathrm{pH}$ 7.3. The column was calibrated with blue dextran $(3 \mathrm{mg} / \mathrm{ml})$ and glucuronolactone $(2 \mathrm{mg} / \mathrm{ml})$ for determination of void volume and total volume, respectively. Fractions were microdialyzed against $150 \mathrm{vol}$ of $200 \mathrm{mM}$ sodium acetate, $0.05 \%$ sodium azide, $\mathrm{pH} 5.5$ for $48 \mathrm{~h}$ at room temperature before assay.

Associative conditions. To determine the aggregatability of PG, HA was added in excess to the extract to ensure maximal reaggregation. Hyaluronic acid (high molecular weight, human umbilical cord, kindly supplied by Dr. P. J. Roughley, Genetics Unit, Shriners Hospital, Mon- treal) at $10 \%(\mathrm{wt} / \mathrm{wt})$ of the PG content (the proteoglycan content in extracts of adult cartilage was estimated as being 6.5 times the total uronic acid (UA) content, which is about $15 \%$ of the total proteoglycan for this age group [39]), was added to the PG extract in $4 \mathrm{M} \mathrm{GuCl}$. The

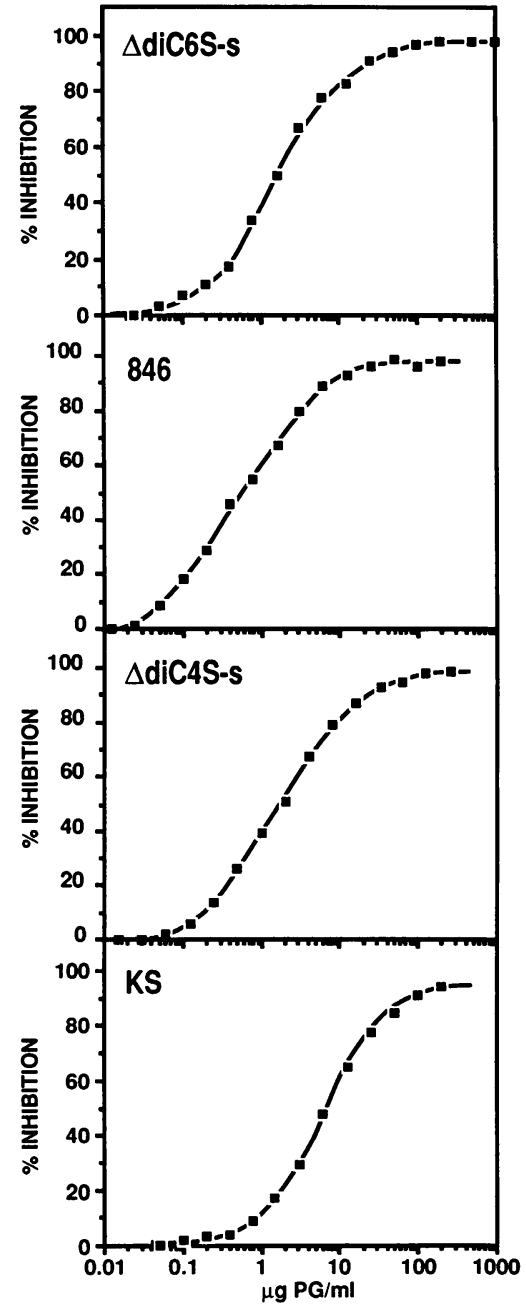

Figure 1. Standard inhibition curves for the RIA of $\triangle$ diC6S-s (antibody $3 \mathrm{~B} 3$ ) and $\Delta$ diC4Ss(antibody 6B) linkage regions to core protein; of CS epitope 846 (antibody 846 ) and of KS (antibody AN9P1). For further details see text and Table I. 
sample was then dialyzed for $24 \mathrm{~h}$ at $4^{\circ} \mathrm{C}$ against $150 \mathrm{vol} 200 \mathrm{mM}$ sodium acetate, $0.05 \%$ sodium azide, $\mathrm{pH}$ 5.5. The dialyzed sample was chromatographed $(500 \mu \mathrm{l})$ on a Sepharose CL-2B column (same dimension as above) and eluted with $200 \mathrm{mM}$ sodium acetate, $0.5 \%$ sodium azide, $0.25 \% \mathrm{BSA}, \mathrm{pH} 5.5$, at the same flow rate and fraction volume as above. Aggregation was recorded as that $P G$ eluting at equal to or less than $\mathrm{Kav}=\mathbf{0 . 1 5}$.

Sepharose CL-6B gel chromatography was used to investigate chain length of CS. Proteoglycan extracts were dialyzed overnight at $4^{\circ} \mathrm{C}$ against $0.2 \mathrm{M}$ acetate buffer, $\mathrm{pH} 5.0$, and then digested in the presence of $5 \mathrm{mM}$ cysteine with $0.2 \mathrm{mg} / \mathrm{ml}$ papain at $37^{\circ} \mathrm{C}$. After $4 \mathrm{~h}$, a further $0.2 \mathrm{mg}$ papain was added. The digestion was terminated after $24 \mathrm{~h}$ by the addition of $10 \mathrm{mM}$ iodoacetamide. Digests $(1 \mathrm{ml})$ were chromatographed on Sepharose CL-6B $(100 \mathrm{~cm} \times 1.25 \mathrm{~cm})$ in $0.2 \mathrm{M}$ acetate buffer, $\mathrm{pH} 5.5$, at a flow rate of $6 \mathrm{ml} / \mathrm{h}$ and fraction vol of $1 \mathrm{ml}$. Chondroitin sulfate was determined by UA analyses.

Statistical analyses. Means \pm SD were recorded for $n$ samples. Student's unpaired $t$ test was used where indicated. $P \leq 0.05$ was considered significant.

\section{Results}

Histology. Histological examination of tissues permitted assignment of a Mankin grade to each of the normal and OA tissues examined. Normal cartilage grades ranged from 0 to 3 , whereas those from OA joints ranged from 2 to 13, according to the degree of degenerative change (Table II). Even within the same specimen grade could vary. Therefore, the grades assigned were representative of the overall changes and indicated the changes shown by the majority of the tissue. The grade assigned was within the range $0-6$ or $7-13$, permitting an arbitrary classification of tissues into early (phase I) (grade 0-6) or advanced (phase II) (grades 7-13) degenerative changes.

Efficiency of proteoglycan extraction. To measure the percentage of PG extracted from frozen-sectioned articular cartilage, UA contents of cartilage extracts and pellets were determined (Table II). The percentage of UA extracted ranged from

Table II. Total Contents of UA, the Different PG Epitopes and HA of Normal and OA Cartilage Extracts

\begin{tabular}{|c|c|c|c|c|c|c|c|}
\hline Sample age & $\begin{array}{c}\text { Mankin } \\
\text { grade }\end{array}$ & $\Delta$ diC6S-s & $\Delta$ diC4S-s & 846 & $\mathrm{KS}$ & $\begin{array}{c}\text { Uronic } \\
\text { acid }\end{array}$ & HA \\
\hline$y r$ & & $\mu g / m g$ wet $w t$ & $\mu g / m g$ wet wt & $n g / m g$ wet wt & $\mu g / m g$ wet wt & $\mu g / m g$ wet $w t$ & $\mu g / m g$ wet $w t$ \\
\hline \multicolumn{8}{|l|}{ Normal } \\
\hline N\#1 47a & 0 & 56 & 0.73 & 12 & 41 & 3.17 & 0.53 \\
\hline $47 b$ & 0 & 78 & 0.63 & 33 & 40 & 4.16 & 0.49 \\
\hline $\mathrm{N \# 2} 73 \mathrm{a}$ & 3 & 36 & 0.87 & 29 & 35 & 2.76 & 0.60 \\
\hline $73 b$ & 1 & 15 & 0.60 & 70 & 18 & 2.52 & 0.58 \\
\hline N\#3 79a & 2 & 17 & 0.72 & 70 & 23 & 3.03 & 0.48 \\
\hline $79 b$ & 3 & 8 & 0.22 & 50 & 5 & 1.75 & 0.48 \\
\hline N\#4 81 & 1 & 56 & 1.10 & 49 & 32 & 3.37 & 0.36 \\
\hline N\#5 79 & 1 & 17 & 1.30 & 103 & 23 & 1.61 & 0.34 \\
\hline N\#6 81 & 2 & 10 & 0.61 & 56 & 23 & 1.71 & 0.40 \\
\hline Mean & & 33 & 0.75 & 52 & 27 & 2.68 & 0.47 \\
\hline SD & & 25 & 0.31 & 27 & 12 & 0.87 & 0.09 \\
\hline \multicolumn{8}{|c|}{$\begin{array}{c}\text { Mankin grade 2-6 } \\
\text { phase I OA }\end{array}$} \\
\hline OA\#1 74a & 2 & 17 & 2.79 & 42 & 44 & 2.99 & 0.45 \\
\hline $74 b$ & 2 & 14 & 5.58 & 108 & 20 & 2.60 & 0.30 \\
\hline OA\#2 70a & 2 & 10 & 6.00 & 261 & 11 & 3.51 & 0.41 \\
\hline OA\#3 74 & 5 & 15 & 1.47 & 243 & 9 & 1.78 & 0.43 \\
\hline OA\#4 74 & 6 & 44 & 0.90 & 108 & 15 & 3.14 & 0.40 \\
\hline Mean & & 20 & $3.35^{*}$ & $152^{*}$ & 20 & 2.80 & $0.40^{*}$ \\
\hline $\mathrm{SD}$ & & 14 & 2.34 & 95 & 14 & 0.66 & 0.06 \\
\hline \multicolumn{8}{|c|}{$\begin{array}{c}\text { Mankin grade } 7-13 \\
\text { phase II OA }\end{array}$} \\
\hline OA\#5 77a & 9 & 45 & 1.51 & 144 & 31 & 3.91 & 0.58 \\
\hline $77 \mathrm{~b}$ & 7 & 10 & 3.20 & 4 & 5 & 2.20 & 0.26 \\
\hline OA\#6 73a & 10 & 11 & 4.50 & 225 & 18 & 2.28 & 0.30 \\
\hline $73 b$ & 8 & 16 & 4.10 & 216 & 25 & 2.78 & 0.30 \\
\hline OA\#7 59a & 13 & 7 & 1.27 & 306 & 15 & 1.37 & 0.26 \\
\hline $59 b$ & 10 & 6 & 1.26 & 324 & 5 & 1.40 & 0.24 \\
\hline OA\#2 70b & 7 & 26 & 2.93 & 261 & 11 & 3.51 & 0.41 \\
\hline Mean & & $17^{*}$ & $2.68^{*}$ & $255^{*}$ & $16^{*}$ & 2.49 & $0.34^{*}$ \\
\hline SD & & 14 & 1.36 & 65 & 10 & 0.98 & 0.12 \\
\hline
\end{tabular}

The OA group is divided into phase I OA (Mankin grade $\leq 6$ ) and phase II OA (Mankin grade $\geq 7$ ). From some joints two specimens were taken $(\mathrm{a}$ and $\mathrm{b})$. * Significant when compared with normals $(P \leq 0.05)$. 
56 to $82 \%$ (data not shown). Compared to normal cartilage (mean 68.6 $\pm 8.7 \mathrm{SD}$ ), the percentage extracted from OA cartilage with grades $\leq 6$ (mean $69.8 \pm 5.4 \mathrm{SD})$ and grades $7-13$ (mean 73.2 $\pm 8.4 \mathrm{SD}$ ) did not change.

Total content of extractable proteoglycan epitopes, uronic acid and $H A$ in normal and osteoarthritic cartilages and Mankin grade. The need to use different PG standards (fetal and adult), prevented a comprehensive analysis of the absolute compositions of the different molecules identified in this study. But these analyses did permit comparisons to be made of the relative content of fetal epitopes ( $\Delta$ diC4S-s and 846 ), adult epitopes ( $\Delta$ diC6S-s and $\mathrm{KS}$ ), and epitope differences between healthy and OA cartilages. There were differences in epitope contents when samples from different sites on the same femoral condyles were examined. But these contents were often recognizably different from those in OA cartilages. In OA cartilages, several trends (Fig. 2) and significant differences (Table II) were observed. UA content, $\triangle$ diC6S-s, KS, and HA contents tended to decrease, whereas $\Delta$ diC4S-s and epitope 846 increased with Mankin grade (Fig. 2). To permit a clearer analysis, these changes in OA were classified as phase I (grades 2-6) or phase II OA (grades 7-12) (Table II). Chondroitin 6-sulfate stubs were significantly decreased in phase II more than in phase I. Keratan sulfate and HA contents were also significantly reduced in phase II more than in phase I when compared with normals. Uronic acid content was not significantly changed in phase I and phase II OA. $\triangle$ diC4S-s and epitope 846 were significantly increased in both phase I and II when compared with normals. These observations clearly reveal compositional changes in OA cartilages that are related to the degree of degeneration revealed by histological grading.
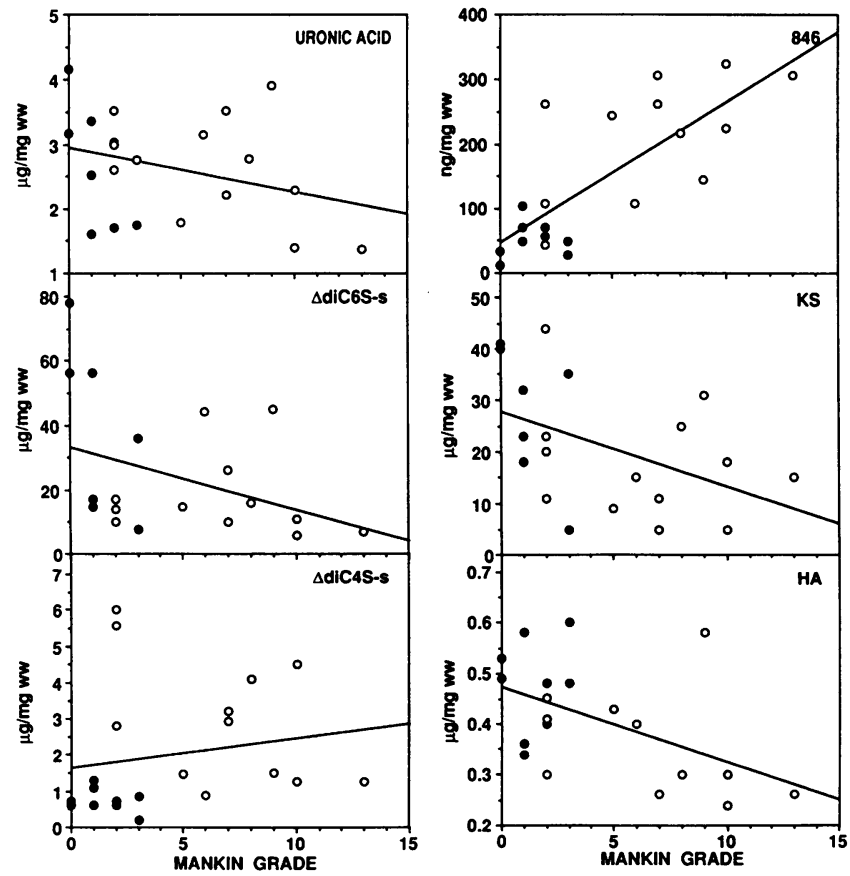

Figure 2. Relationship of content of PG epitopes and HA to Mankin grade. Values for normal $(\bullet)$ and OA samples $(O)$ are indicated. To indicate general trends the best fit lines are shown which are derived from regression analyses of the all values. Since analyses were made of both normal and OA samples regression analyses cannot be made.
A

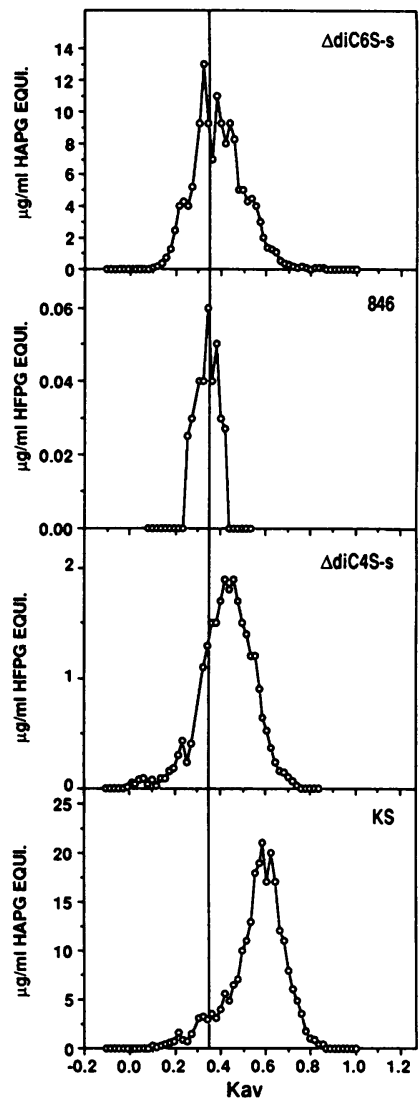

B

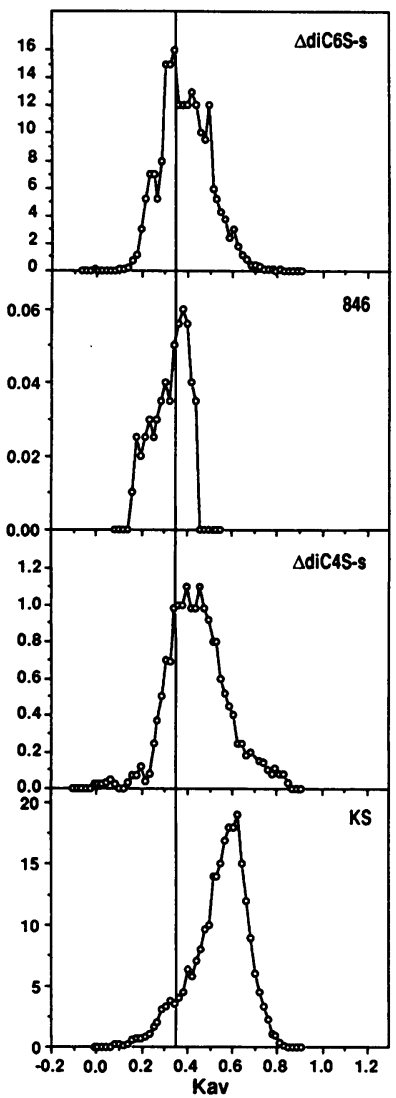

Figure 3. Gel chromatography of aggrecan PG populations from normal cartilages on Sepharose CL-2B under dissociative conditions (with $4 \mathrm{M} \mathrm{GuCl}$ ) to demonstrate heterogeneity of aggrecan in a 81yr-old, grade $1(A)$ and a 79-yr-old grade $2(B)$ are shown.

Identification of different proteoglycan populations in normal and osteoarthritic cartilages using dissociative (4MGuCl) and associative chromatography on Sepharose $C L-2 B$

The percentage recovery of $\mathrm{PG}$ epitopes from chromatography under either conditions ranged from 70 to $85 \%$.

Monomer size. Column fractions of chromatography under dissociative conditions were assayed with each antibody to investigate monomer size, and the results were expressed as intact PG equivalents. The chromatograms for representative normal samples are shown in Fig. 3, and representative OA samples are shown in Fig. 4. These analyses revealed the presence of different populations of PG in normal and OA cartilages characterized by differences in composition and size. For example, in normal cartilage, the largest population eluted at a $\mathrm{Kav} \sim 0.35$. It was recognized using the anti- $\Delta$ diC6S-s antibody. Eluting at approximately the same Kav, and present in a relatively very small amount was a population bearing epitope 846 . Slightly smaller than the $\Delta$ diC6S-s population was a population eluting at $\mathrm{Kav} \sim 0.45$. This was recognized by the anti$\Delta$ diC4S-s antibody, 6B. This suggests that the $\triangle \mathrm{diC} 4 \mathrm{~S}$-s population is smaller than and distinct from the $\triangle$ diC6S-s and epitope 846 population(s). Eluting at $\mathrm{Kav} \sim 0.6$ was a $\mathrm{PG}$ population recognized by the anti-KS antibody, AN9P1. This is the small KS-rich PG population previously described by us (27) and 


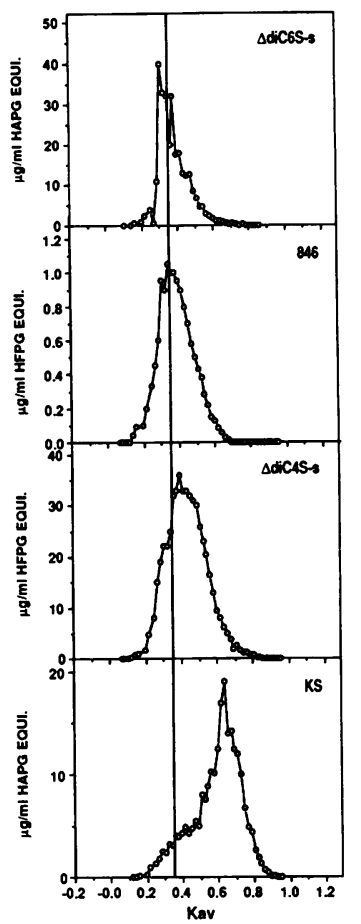

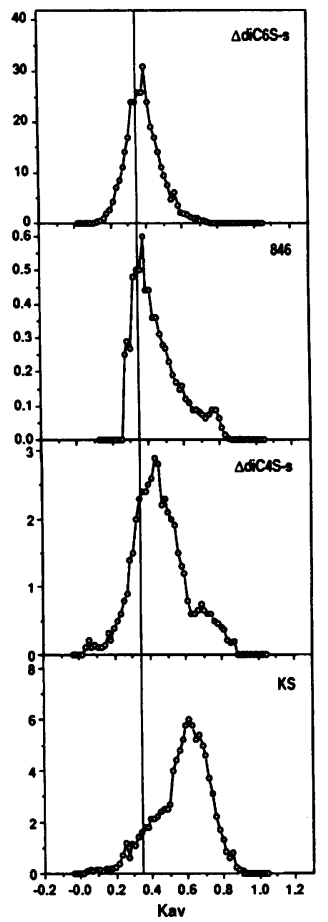

c

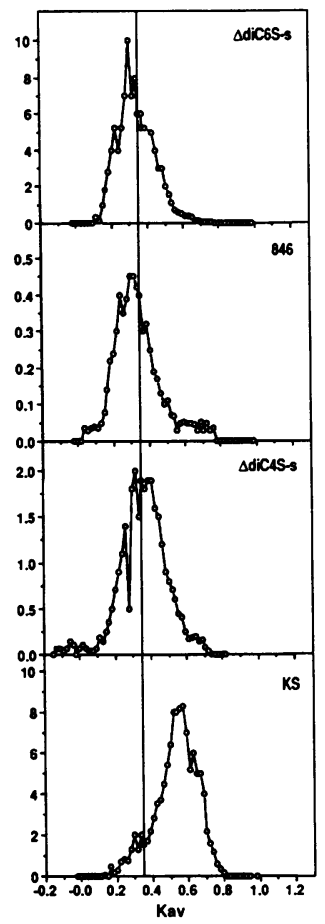

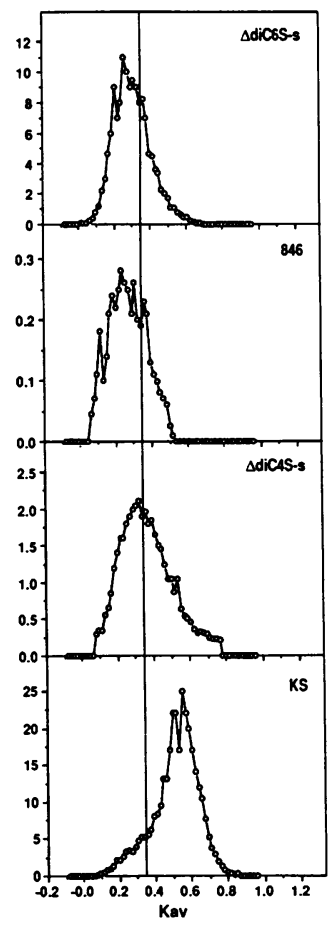

Figure 4. Gel chromatography of aggrecan populations from OA cartilages on Sepharose CL-2B under dissociative conditions to demonstrate heterogeneity of aggrecan in phase $\mathrm{I}:(A)$ a 70-yr-old, grade $2 ;(B)$ 74-yrold grade 5 ; and phase II: $(C)$ a 59 -yr-old, grade $13 ;(D)$ a 73 -yr-old, grade 9.

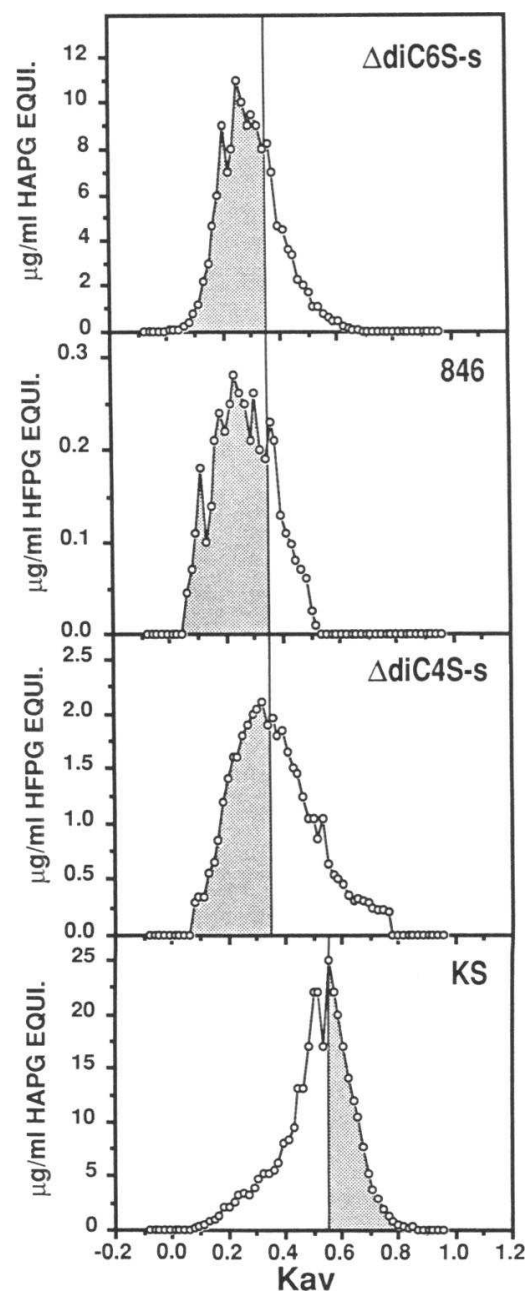

Figure 5. Method of determination of relative hydrodynamic sizes of aggrecan populations by assigning arbitrary Kav values, above or below which the percentage of the total content in the shaded area was recorded. The example given is OA sample 73-yr-old, grade 10. recognized as a degradation product of aggrecan containing the $\mathrm{KS}$-rich region. In general, the elution for $\Delta$ diC6S, $\Delta$ diC4S, and 846 was often but not always unimodal. A small "shoulder" of higher molecular weight PG was seen in the elution profile for KS. Similar populations were identified in OA cartilages, irrespective of grade.

To compare these populations in normal and OA cartilages, the following approach was used as outlined in Fig. 5. The relative contents of epitopes in column fractions less or greater than a given Kav, which was fixed for each epitope in normal and OA cartilages, were determined and expressed as a percentage of the total epitope content in all column fractions. Therefore, for epitopes $\Delta$ diC6S-s, 846, and $\Delta$ diC4S-s (Kav $\leq 0.35)$ and for $\mathrm{KS}(\mathrm{Kav} \geq 0.55)$, relative hydrodynamic sizes were calculated as percentages. These results are shown in Table III. The data shown are therefore for the percentage amount of a PG of a given hydrodynamic size. Examination of the different populations in normal cartilages confirmed the presence of two populations of similar size containing $\Delta$ diC6S-s and 846 epitopes. These were significantly larger than the population containing $\Delta$ diC4S-s (Table III). The presence of a much smaller KS-rich population is again apparent.

When the sizes of the different PG in all the OA samples were compared with the normal samples, there was no evidence for any significant differences. But when the OA specimens were divided into two groups by their grades, phase I (grade $\leq 6$ ) and phase II (grades $\geq 7$ ), significant differences were observed ( Table III). The equivalent PG proteoglycans in phase I OA were generally smaller than in normal cartilage but the differences were not significant. But in phase II OA, molecules bearing epitopes $\Delta$ diC6S-s, 846 , and $\Delta$ diC4S-s were significantly larger in size than the corresponding PG in phase I OA (grades 7-13). The predominant small KS-rich PG species showed no significant changes ( Table III), although there was a 
Table III. Comparison of Relative Hydrodynamic Sizes of Different Aggrecan Populations in Normal, Phase I, and Phase II OA, Determined as Shown in Fig. 5

\begin{tabular}{llc}
\hline $\begin{array}{c}\text { Tissue and } \\
\text { epitope (Kav) }\end{array}$ & $\begin{array}{c}\text { Epitope } \\
\text { mean } \pm \text { SD } \\
(\%)\end{array}$ & $\begin{array}{c}\text { Significance } \\
P \text { values }\end{array}$ \\
\hline $\begin{array}{l}\text { diC6S-s } \leq 0.35 \\
\text { Normals }\end{array}$ & $53.0 \pm 12.7$ & \\
Phase I OA & $40.7 \pm 12.7$ & 0.1889 \\
Phase II OA & $62.4 \pm 12.6$ & $0.0372^{*}$ \\
$846 \leq 0.35$ & & \\
$\quad$ Normals & $47.2 \pm 11.5$ & 0.0551 \\
Phase I OA & $32.3 \pm 6.4$ & $0.0145^{*}$ \\
Phase II OA & $62.4 \pm 17.8$ & 0.1916 \\
$\Delta$ diC4S-s $\leq 0.35$ & $31.4 \pm 9.6$ & $0.0121^{*}$ \\
$\quad \begin{array}{l}\text { Normals } \\
\text { Phase I OA } \\
\text { Phase II OA }\end{array}$ & $24.3 \pm 1.5$ & \\
KS $\geq 0.55$ & $44.2 \pm 11.6$ & 0.1114 \\
Normals & & 0.1297 \\
Phase I OA & $50.6 \pm 4.7$ & \\
Phase II OA & $58.2 \pm 7.8$ & \\
\hline
\end{tabular}

$P$ values are shown indicating significant differences $\left({ }^{*}\right)$ between phase I (Mankin grades 2-6) and phase II (Mankin grades 7-13) OA. Five normal, four phase I, and five phase II samples were analyzed. In addition to the statistical analyses indicated, analyses of normal populations rich in CA were made: 846 was not significantly smaller in size than $\Delta$ diC6S-s $(P=0.4708)$, but $\Delta$ diC4S-s was significantly different in size than $846(P=0.0461)$ and $\Delta$ diC6S-s $(P=0.0162)$.

trend towards a decrease in size in phase I OA and an increase in size in phase II OA.

Chondroitin sulfate chain length. To determine whether these changes in PG monomer size resulted from a change in synthesis resulting in altered CS chain length, $\mathrm{PG}$ were digested with papain and chromatographed under associative conditions on Sepharose CL-6B and analyzed for UA. The results are shown in Fig. 6. There was no evidence for any change in chain length in OA cartilage of various Mankin grades.

Monomer aggregation. Analysis of these populations under associative conditions revealed that in normal cartilages, between 50 and $59 \%$ of each of these populations aggregated with the exception of the population identified by epitope 846 , which showed almost complete aggregation (Table IV, Fig. 7). Therefore, there exist two populations of the largest size, one identifiable by the $\Delta$ diC6S-s stubs on core protein and a more minor population bearing epitope 846 .

Analysis of aggregation of PG from OA cartilages with $\mathrm{HA}$ revealed similar aggregation for $\triangle$ diC6S-S and KS epitopes to that seen in normal cartilage (Table IV). But epitopes 846 (phase I and II) and $\Delta$ diC4S-s (phase II only) exhibited much less aggregation in OA cartilage.

\section{Discussion}

By using immunochemical methods to study PG in total cartilage extracts we have characterized recognizably different ag-

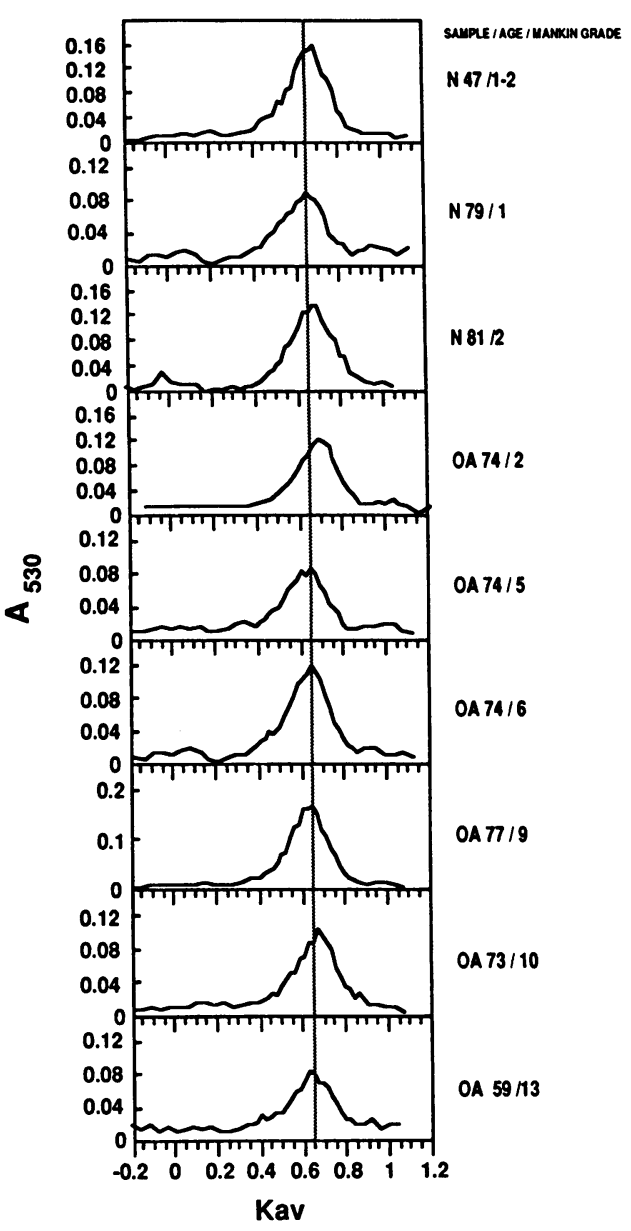

Figure 6. Analysis on Sepharose CL-6B of hydrodynamic sizes of CS chains in normal $(N)$ and $O A$ cartilages. The age and grade of the sample is indicated.

Table IV. Percentage Aggregation of Proteoglycans Bearing the Epitope Indicated

\begin{tabular}{lcccc}
\hline & \multicolumn{4}{c}{ Epitopes } \\
\cline { 2 - 5 } & $\Delta$ diC6S-s & KS & $\Delta$ diC4S-s & 846 \\
\hline Normals & & & & \\
Mean & 59.0 & 50.0 & 52.0 & 96.1 \\
SD & \pm 12.6 & \pm 10.0 & \pm 5.3 & \pm 3.5 \\
& $(n=5)$ & $(n=5)$ & $(n=4)$ & $(n=6)$ \\
Phase I OA & & & & \\
$\quad$ (grades 2-6) & & & & \\
Mean & 65.0 & 61.3 & 49.0 & 58.3 \\
SD= & \pm 6.9 & \pm 4.9 & \pm 7.0 & \pm 7.6 \\
& $(n=4)$ & $(n=3)$ & $(n=2)$ & $(n=3)$ \\
Phase II OA & & & & \\
$\quad$ grades 7-13) & & & & \\
Mean & 56.0 & 48.7 & 33.5 & 58.5 \\
SD= & \pm 6.0 & \pm 9.6 & \pm 2.1 & \pm 13.4 \\
& $(n=3)$ & $(n=4)$ & $(n=2)$ & $(n=2)$ \\
& & & & \\
\hline
\end{tabular}

Aggregation was measured as that PG eluting at $\leq \mathrm{Kav}=0.15$. 


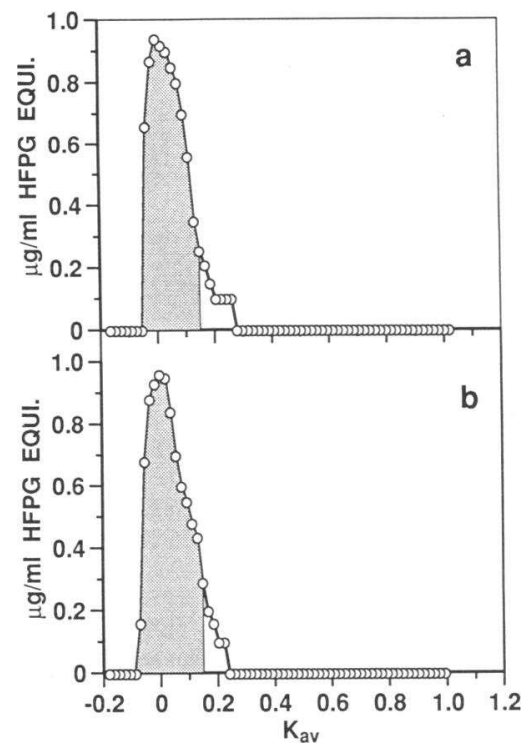

Figure 7. Gel chromatography on Sepharose CL-2B under associative conditions in the presence of exogenous HA of aggrecan populations from two normal cartilages to demonstrate aggregation of epitope 846-containing molecules with hyaluronic acid in $(a)$ a 49-yr-old female and $(b)$ a 54-yrold male. Percent of aggregation (shaded area) is $(a) 91.8$ and $(b) 92.1$. Recoveries from column fractions of added samples were $(a) 112 \%$ and $(b) 92 \%$.

grecan populations in normal and OA cartilages. The previously described smaller KS-rich molecules $(27,29,41)$ are thought to represent degradation products derived from the larger populations that are retained in cartilage (in part at least) as part of their aggregation with hyaluronic acid. In addition, larger $\Delta$ diCS-s-rich populations were observed which have not previously been described. Proteoglycans bearing $\Delta$ diC6S-s (the linkage region of CS to core protein) were larger than those in which $\triangle$ diC4S-S was the predominant "linkage" sulfation. Molecules bearing epitope 846 (a very minor population compared with those bearing $\mathrm{C} 4 \mathrm{~S}$ ( the same standard was used for these two assays) cochromatographed with PG-bearing $\Delta$ diC6S-s. But in normal cartilages, molecules bearing epitope 846 showed almost complete aggregation with HA, unlike those bearing $\Delta$ diC6S-s. This indicated the presence of four recognizable populations: two of similar large size, an intermediate size $\Delta$ diC4S-S-rich population, and the small KS-rich molecules. These differences in the large molecules may also result from different dominant CS substitutions, as suggested by earlier studies from many laboratories.

This heterogeneity displayed by these larger molecules, revealed by the use of antibodies, was not previously detectable by the traditional uronic acid or hexosamine analyses (32-35, 41 ). It could result in part from differences in sulfation of CS linkage regions along the CS-attachment zone. The recognition of two distinct domains in the core protein of the CS-attachment region of human aggrecan (13) has led to the assignment of two adjacent CS domains, CS1 and CS-2, the latter being closest to the G3 globular domain. It is conceivable that the $\Delta$ diC6S-s and $\Delta$ diC4S-s may be found predominantly in the CS2 and CS1 domains, respectively. Thus, if all or part of the CS2 domain was removed by proteolysis in the extracellular matrix, this would result in a smaller molecule enriched in the CS1 domain and $\Delta$ diC4S-s linkages. In normal cartilage, molecules bearing epitope 846 exhibit essentially complete aggregation with HA. Few nonaggregating molecules were observed. This was due to lack of sensitivity of the immunoassay. It is possible, therefore, in view of their large size and complete aggregation, that molecules bearing epitope 846 represent re- cently synthesized molecules. If these epitopes are present on the same molecule, the very low content of epitope 846 relative to $\Delta$ diC4S-s suggests that the CS chains bearing this epitope may be in a part of the molecule that is readily removed from PG in the extracellular matrix: This would arguably be close to the $\mathrm{G} 3$ domain ( since there is no evidence for CS in the $\mathrm{G} 1$ and G2 domains). Alternatively, epitope 846 may be present on a distinct very minor population of PG. Clearly further careful experimental work is required to investigate further the structures and heterogeneity of these molecules.

In any study of articular cartilages in OA, it is extremely important to take note of the wide variation in degenerative changes that are a feature of this disease. Although it is not perfect, the grading system introduced by Mankin et al. has permitted the identification of compositional and biosynthetic changes in PG in OA. Thus, in early disease (low grade) synthesis of PG was found to be increased, whereas in advanced disease it was decreased $(37,38)$. This loss of synthesis accompanied a net loss of PG that was only clearly observed in advanced disease. Using this approach, we examined PG size, composition, aggregation, and content as the disease progresses.

All our PG populations were observed in OA cartilage irrespective of grade. However, the aggregation differences between the populations bearing epitope 846 and $\Delta$ diC6S-s were no longer observed. Changes were noted in these two epitopes indicative of changes in CS composition. $\Delta$ diC6S-s content was reduced, whereas epitope 846 and $\triangle \mathrm{diC} 4 \mathrm{~S}$-s contents were increased. This probably occurred when there was no overall change in uronic acid content, although KS content was reduced. The assignment of two phases of OA permitted the clear recognition of pathology-related changes that have not previously been reported. In phase I, there is evidence for a general reduction (although not significant) in the sizes of all the PG populations compared to their normal counterparts. Since this is accompanied by the loss of $\Delta$ diC6S-s, this may indicate the loss of the G3 and CS2 domains, according to our proposed assignment of $\triangle \mathrm{diC} 6 \mathrm{~S}-\mathrm{s}$ to the CS2 domain. This reduction in size was not accompanied by a change in CS chain length, suggesting that increased proteolysis occurred involving the core protein. Moreover, the loss of aggregation shown by the population bearing epitope 846 is also indicative of proteolysis.

In phase II of the disease (grades 7-13), when fibrillation is more extensive and cartilage becomes very degenerate in appearance, the CS-rich and KS-rich PG populations were still detected. But they were all larger than their normal counterparts and (except for the KS-rich molecules) were significantly larger than their phase I counterparts. These increases in size did not result from changes in CS chain length, (observed previously, reference 42 ), suggesting that these larger molecules contained longer core proteins and were less degraded.

Collectively, these observations indicate that in $\mathrm{OA}$, two phases can be identified in the pathological changes involving aggrecan that occur in the articular cartilages. The initial phase I is characterized by a general degradation of PG molecules combined with replacement with new molecules of altered composition, as indicated earlier $(37,38)$. Then in phase II there is extensive replacement of degraded molecules with larger compositionally different molecules, probably as a result of the increased synthesis described previously. Evidence for changes in the biosynthesis of CS come not only from our studies of $\Delta$ diC4S-s and $\Delta$ diC6S-s but also from the increase in 
content of the native CS epitope 846: The latter would be expected if, as we suggest, epitope 846 is present on recently synthesized molecules and is located on CS close to the G3 domain. A similar increase in content of another native CS epitope recognized by monoclonal antibody $3 \mathrm{~B} 3$ has also been observed in experimental OA in dogs (43). Since these native CS epitopes are most commonly found in fetal and embryonic tissues, their reappearance in OA suggests that changes in the environment of the chondrocyte cause changes in synthesis and CS assembly.

Eventually, as a result of extensive damage to the cartilage, PG is lost. This probably results from extensive damage to the collagen fibrillar organization we described previously (44) leading to the loss of HA $(45,46)$ and of $\operatorname{PG~}(37,38)$ observed in the present study as well as previously. But, contrary to common belief, before this happens, our observations indicate that OA cartilage has a considerable capacity for extensive turnover of the PG component of the extracellular matrix. If collagen damage could be prevented and its repair promoted then it may be possible for cartilage to repair itself.

\section{Acknowledgments}

We thank Renée Leiberman and Audrey Wheeler, who processed this manuscript, and Mark Lepik and Jane Wishart, who prepared the figures.

The study was funded by the Medical Research Council of Canada and the Shriners of North America (to Dr. A. Robin Poole).

\section{References}

1. Sokoloff, L., and A. J. Hough, Jr. 1985. Pathology of osteoarthritis. In Arthritis and Allied Conditions. A Textbook of Rheumatology. 10th ed. D. J. McCarty, editor. Lea and Febiger, Philadelphia. 1377-1399.

2. Kempson, G. E. 1979. Mechanical properties of articular cartilage. In Adult Articular Cartilage, M. A. R. Freeman, editor. Pitman Books Ltd. Tunbridge Wells, Kent, England. 333-414.

3. Mow, V. C., D. S. Howell, and J. A. Buckwalter. 1990. Structure and function relationships of articular cartilage and the effects of joint instability and trauma on cartilage function. In Cartilage Changes in Osteoarthritis. K. D. Brandt, editor. Indiana University School of Medicine, Indianapolis, IN. 22-42.

4. Mendler, M., S. G. Eich-Bender, L. Vaughan, K. Winterhalter, and P. Bruckner. 1989. Cartilage contains mixed fibrils of collagen types II, IX, and XI. J. Cell Biol. 108:191-197.

5. Eyre, D. R., S. Apone, J. J. Wu, L. H. Ericsson, and K. A. Walsh. 1987. Collagen type IX: evidence for covalent linkages to type II collagen in cartilage. FEBS (Fed. Eur. Biochem. Soc.) Lett. 220:337-341.

6. van der Rest, M., and R. Mayne. 1988. Type IX collagen proteoglycan from cartilage is covalently cross-linked to type II collagen. J. Biol. Chem. 263:6151618.

7. Poole, A. R., I. Pidoux, A. Reiner, and L. Rosenberg. 1982. An immunoelectron microscope study of the organization of proteoglycan monomer, link protein, and collagen in the matrix of articular cartilage. J. Cell Biol. 93:921-937.

8. Heinegård, D., and V. C. Hascall. 1974. Aggregation of cartilage proteoglycans. III. Characteristics of the proteins isolated from trypsin digest of aggregates. J. Biol. Chem. 249:4250-4256.

9. Tang, L-H., L. Rosenberg, A. Reiner, and A. R. Poole. 1979. Proteoglycans from bovine nasal cartilage. Properties of a soluble form of link protein. J. Biol. Chem. 254:10523-10531.

10. Mow, V. C., W. Zhu, W. M. Lai, T. E. Hardingham, C. Hughes, and H. Muir. 1989. The influence of link protein stabilization on the viscometric properties of proteoglycan aggregate solutions. Biochim. Biophys. Acta 992:201-208.

11. Neame, P. J., J. E. Christner, and J. R. Baker. 1987. Cartilage proteoglycan aggregates. The link protein and proteoglycan amino-terminal globular domains have similar structures. J. Biol. Chem. 262:17768-17778.
12. Doege, K., M. Sasaki, E. Horigan, J. R. Hassell, and Y. Yamada. 1987. Complete primary structure of the rat cartilage proteoglycan core protein deduced from cDNA clones. J. Biol. Chem. 262:17757-17767.

13. Doege, K. J., M. Sasaki, T. Kimura, and Y. Yamada. 1991. Complete coding sequence and deduced primary structure of the human cartilage large aggregating proteoglycan aggrecan. Human-specific repeats and additional alternatively spliced forms. J. Biol. Chem. 266:894-902.

14. Antonsson, P., D. Heinegård, and A. Oldberg. 1989. The keratan sulfateenriched region of bovine cartilage proteoglycan consists of a consecutively repeated hexapeptide motif. J. Biol. Chem. 264:16170-16173.

15. Halberg, D. F., G. Proulx, K. Doege, Y. Yamada, and K. Drickamer. 1988. A segment of the cartilage proteoglycan core protein has lectin-like activity. J. Biol. Chem. 263:9486-9490.

16. Caterson, B., J. E. Christner, J. R. Baker, and J. R. Couchman. 1985. Production and characterization of monoclonal antibodies directed against connective tissue proteoglycans. Fed. Proc. 44:386-393.

17. Matsui, Y., M. Alini, C. Webber, and A. R. Poole. 1991. Characterization of aggregating proteoglycans from the proliferative, maturing, hypertrophic and calcifying zones of the cartilage physis (growth plate). J. Bone Jt. Surg. 73A:1064-1074.

18. Glant, T. T., K. Mikecz, P. J. Roughley, E. Buzas, and A. R. Poole. 1986. Age-related changes in protein-related epitopes of human articular cartilage proteoglycans. Biochem. J. 236:71-75.

19. Sorrell, J. M., A. M. Lintala, F. Mahmoodian, and B. Caterson. 1988. Epitope specific changes in chondroitin sulfate/dermatan sulfate proteoglycans as markers in the lymphopoietic and granulopoietic compartments of developing bursae of Fabricus. J. Immunol. 140:4263-4270.

20. Caterson, B., J. E. Christner, and J. R. Baker. 1983. Identification of a monoclonal antibody that specifically recognizes corneal and skeletal keratan sulfate. J. Biol. Chem. 258:8848-8854.

21. Poole, A. R., C. Webber, A. Reiner, and P. J. Roughley. 1989. Studies of a monoclonal antibody to skeletal keratan sulfate. Importance of antibody valency. Biochem. J. 260:849-856.

22. Mehmet, H., P. Scudder, P. W. Tang, E. F. Hounsell, B. Caterson, and T. Feizi. 1986. The antigenic determinants recognized by three monoclonal antibodies to keratan sulfate involve sulphated hepta or larger oligosaccharides of the poly-( $N$-acetylactosamine) series. Eur. J. Biochem. 157:385-391.

23. Maroudas, A. 1980. Physical chemistry of articular cartilage and the intervertebral disc. In The Joints and Synovial Fluid. Vol. II. L. Sokoloff, editor. Academic Press, New York. 239-291.

24. Kempson, G. E., H. Muir, M. A. R. Freeman, and S. A. V. Swanson. 1970. Correlations between the compressive stiffness and chemical constituents of human articular cartilage. Biochim. Biophys. Acta. 215:70-77.

25. Lai, W. M., J. S. Hou, and V. C. Mow. 1991. A biphasic theory of the swelling and deformation behaviours of articular cartilage. J. Biomech. Eng. 113:245-258.

26. Roughley, P. J., R. J. White, and A. R. Poole. 1985. Identification of a hyaluronic acid protein that interferes with the preparation of high-buoyant density proteoglycan aggregates from adult human articular cartilage. Biochem. $J$. 231:129-138.

27. Webber, C., T. T. Glant, P. J. Roughley, and A. R. Poole. 1987. The identification and characterization of two populations of aggregating proteoglycans of high buoyant density isolated from post-natal human articular cartilages of different ages. Biochem. J. 248:735-740.

28. Franzen, A., S. Inerot, S-O. Hejderup, and D. Heinegård. 1981. Variations in the composition of bovine hip articular cartilage with distance from the articular surface. Biochem. J. 195:535-543.

29. Bayliss, M. T., and S. Y. Ali. 1978. Age-related changes in the composition and structure of human articular cartilage proteoglycans. Biochem. J. 176:683693.

30. Mort, J. S., A. R. Poole, and P. J. Roughley. 1983. Age-related changes in the structure of proteoglycan link protein in normal human articular cartilage. Biochem. J. 214:269-272.

31. Nguyen, Q., G. Murphy, P. J. Roughley, and J. S. Mort. 1989. Degradation of proteoglycan aggregate by a cartilage metalloproteinase. Biochem. $J$. 259:61-67.

32. Brocklehurst, R., M. T. Bayliss, A. Maroudas, H. L. Coysh, M. A. R. Freeman, P. A. Revell, and S. Y. Ali. 1984. The composition of normal and osteoarthritic articular cartilage from human knee joints. J. Bone Jt. Surg. Am. Vol. 66A:95-106.

33. Sweet, M. B. E., E. J-M. A. Thonar, A. R. Immelman, and L. Solomon. 1977. Biochemical changes in progressive osteoarthritis. Ann. Rheum. Dis. 36:387-398.

34. Vasan, N. 1980. Proteoglycans in normal and severely osteoarthritic human cartilage. Biochem. J. 187:781-787.

35. Bayliss, M. T., and S. Y. Ali. 1978. Isolation of proteoglycans from human articular cartilage. Biochem. J. 169:123-132. 
36. Palmoski, M., and K. Brandt. 1976. Hyaluronate-binding by proteoglycans: comparison of mildly and severely osteoarthritic regions of human femoral cartilage. Clin. Chim. Acta. 70:87-95.

37. Thompson, R. C., and T. R. Oegema, Jr. 1979. Metabolic activity of articular cartilage in osteoarthritis. J. Bone Jt. Surg. 61 A:407-416.

38. Mankin, H. J., H. Dorfman, L. Lippiello, and A. Zarins. 1971. Biochemical and metabolic abnormalities in articular cartilage from osteo-arthritic human hips. II. Correlation of morphology with biochemical and metabolic data. J. Bone Jt. Surg. Am. Vol. 53A:523-537.

39. Roughley, P. J., and R. J. White. 1980. Age-related changes in the structure of the proteoglycan subunits from human articular cartilage. J. Biol. Chem. 255:217-224.

40. Bitter, T., and H. M. Muir. 1962. A modified uronic acid carbazole reaction. Anal. Biochem. 4:330-334.

41. Bayliss, M. T., M. Venn, A. Maroudas, and S. Y. Ali. 1983. Structure of proteoglycans from different layers of human articular cartilage. Biochem. J. 209:387-400.
42. Bollet, A. J., and J. L. Nance. 1966. Biochemical findings in normal and osteoarthritic articular cartilage. II. Chondroitin sulfate concentration and chain length, water and ash content. J. Clin. Invest. 45:1170-1177.

43. Caterson, B. G., F. Mahmoodian, J. M. Sorrell, T. B. Hardingham, M. T. Bayliss, S. L. Carney, A. Ratcliffe, and H. Muir. 1990. Modulation of native chondroitin sulphate structure in tissue development and in disease. J. Cell Science. 97:411-417.

44. Dodge, G. R., and A. R. Poole. 1989. Immunohistochemical detection and immunochemical analysis of type II collagen degradation in human normal, rheumatoid, and osteoarthritic articular cartilages and in explants of bovine articular cartilage cultured with Interleukin 1. J. Clin. Invest. 83:647-661.

45. Thonar, E. J-M. A., M. B. E. Sweet, A. R. Immelman, and G. Lyons. 1978. Hyaluronate in articular cartilage:age-related changes. Calcif. Tissue Res. 26:1921.

46. Sweet, M. B. E., E. J.-M. A. Thonar, A. R. Immelman, and L. Solomon. 1977. Biochemical changes in progressive osteoarthritis. Ann. Rheum. Dis. 36:387-398. 